\title{
Atmospheric Dynamics and Dust-Driven Winds of Carbon Stars
}

\author{
SUSANNE HÖFNER and ERNST A. DORFI \\ Institut für Astronomie der Universität Wien, Vienna, Austria
}

\begin{abstract}
We have calculated radiation-hydrodynamical models of the atmospheres and circumstellar dust shells of C-rich AGB stars which include a time-dependent description of the dust formation process. Simulating the stellar pulsation by a variable inner boundary, we have investigated the timedependent dynamics of the atmosphere and circumstellar envelope and its interaction with dust formation. We have varied the physical parameters of the models systematically to study their influence on the time-dependent behavior and time-averaged mass-loss characteristics of our models. The results can be summarized as follows: The dependence of the mass-loss rate on stellar parameters predicts a strong increase of mass loss as stars evolve along the AGB. As the stellar luminosity increases, while simultaneously the mass and effective temperature decrease, the atmosphere becomes more extended and conditions become more favorable for dust formation and higher densities in the acceleration region of the wind. The models agree nicely with mean mass loss - period relations deduced from observations of Mira stars, and the observed scatter of mass-loss rates for a given period can be understood in terms of pulsation amplitude or non-linearities of the dust condensation and wind mechanism. As expected from radiation-driven outflows, the wind velocities show a good correlation with a quantity characterizing the strength of radiation pressure on dust relative to gravitation.

This paper has appeared in $A \& A 319,648,1997$. Synthetic spectra and IRAS colors based on these models have been calculated by Loidl et al. and by Windsteig et al. (this volume). This work is supported by the Fonds zur Förderung der wissenschaftlichen Forschung (FWF) under project number S7305-AST.
\end{abstract}

